\title{
Sozialkommunikatives Verhalten in klinischer Perspektive
}

\author{
H. Ellgring und D. Ploog
}

\section{Überblick}

In diesem Beitrag soll zunächst versucht werden, einige Besonderheiten von sozial wirksamen nonverbalen Verhaltensweisen darzustellen. Diese Besonderheiten begründen zwar einige methodische Schwierigkeiten in der Erfassung und Interpretation solcher Verhaltensweisen, machen sie aber gleichzeitig auch klinisch interessant. Aus den Beschreibungen des Affektausdrucks bei hirnorganischen Veränderungen geht hervor, daß diese Verhaltensweisen wichtige Informationen über die Störungen und deren Restitution liefern können. Den plastischen klinischen Beschreibungen stehen allerdings nur wenige systematische Untersuchungen gegenüber. Dies beruht wahrscheinlich u.a. darauf, daß bisher wenige überprüfte Methoden für die Analyse nonverbaler Verhaltensweisen verfügbar waren.

Anhand der Veränderungen des nonverbalen Verhaltens bei primär affektiven Störungen, $\mathrm{d}$. h. bei endogen und neurotisch depressiven Erkrankungen sollen solche Methoden dargestellt werden. Die Ergebnisse weisen auf eine Individuenspezifität nonverbaler Reaktionsformen bei affektiven Störungen hin, wonach nur ein Teil des verfügbaren Verhaltensrepertoires sich zustandsabhängig verändert.

Für die Analyse des nonverbalen Verhaltens folgt, daß bei Beurteilungen und Veränderungsmessungen intraindividuelle Verläufe der Störung oder der Krankheit zu berücksichtigen sind. Bei hirnorganischen Störungen sind zudem ausdrucksmotorische und affektive Steuerung zu differenzieren, die wie bei der Parkinson-Erkrankung dissoziiert sein können. Als unmittelbarer, jedoch nicht-invasiver Zugang ist sozialkommunikatives Verhalten als Informationsquelle zu betrachten, aus der quantitative Größen gewonnen werden können, für die sich enge Beziehungen zu veränderlichen affektiven und appetitiven Zuständen nachweisen lassen.

\section{Ausdrucksverhalten und seine Funktionen}

Von den sozialkommunikativen Verhaltensweisen des Menschen sollen hier die nonverbalen Elemente betrachtet werden, d.h. Verhaltensweisen wie Mimik, Gestik, Blickzuwendung etc. Diese Verhaltensweisen, traditionell auch als Ausdrucksverhalten bezeichnet, spielen für die menschliche Verständigung insofern eine wichtige Rolle, als sich in ihnen ein erheblicher Teil der affektiven und kognitiven Vorgänge für den Interaktions- 
partner erkennbar vermittelt. Nonverbales Verhalten gewinnt zusätzlich an Bedeutung, wenn, aus was für Gründen auch immer, die sprech-sprachliche Verständigung erschwert ist oder entfällt. Dies ist bei Gehörlosen evident; aber auch bei älteren Menschen verlagert sich z. B. in der sozialen Interaktion das Gewicht deutlich zugunsten der nonverbalen Elemente in der Verständigung (BLAZER 1982, S. 124; CAPORAEL 1981).

Für die augenblickliche Diskussion sollen kurz einige Besonderheiten erwähnt werden. Nonverbales Verhalten setzt sich aus angeborenen und erworbenen Teilen zusammen, es ist multifunktional und es ist sozial wirksam.

\section{Angeborene und erworbene Anteile}

Ein mimischer Ausdruck, wie z. B. das Lächeln, ist ein angeborenes Bewegungsmuster, ein soziales Signal. Erworben wurde in der Ontogenese wann, wo und wem gegenüber dieses Lächeln gezeigt wird, d.h. wie das verfügbare Repertoire verwendet wird.

Es ist weiterhin anzunehmen, daß man im Laufe der Ontogenese kaum neue Ausdrucksformen erlernt, sondern daß vielmehr der mimische Ausdruck unter willkürliche Kontrolle gelangt. Die Ausdrucksformen nehmen dadurch an Komplexität zu und gewinnen an Differenzierung.

In der mimischen Aktion kann somit gleichzeitig ein Antriebszustand an den Verhaltenseffektoren als auch die bewußte Kontrolle und Darstellung eines Ausdrucks wirksam werden. So drückt die Mimik den Affekt aus; gleichzeitig enthält sie aber auch bewußt gesteuerte Darstellungen und Mitteilungen, die durch die soziale Umgebung und soziale Regeln mitbestimmt sind. Ein Problem bei der Untersuchung solcher Phänomene ist, daß bewußt gesteuerte Anteile und weniger bewußter, unmittelbarer Ausdruck in der Mimik nur schwer von einem Beobachter zu trennen sind. Auch vom Mitteilungsempfänger und Kommunikationspartner werden sie nicht bewußt unterschieden.

Die für das hirnorganische Psychosyndrom beschriebene „Affektinkontinenz" weist z. B. auf die Auflösung der bisher vorhandenen Kontrolle des Affektausdrucks hin. Folgt man der "facial feedback“-Hypothese des Ausdrucks (TomKINs 1962), so könnte dieser unkontrollierte Ausdruck in einer Rückkoppelung zu einer Verstärkung des erlebten Gefühlszustandes beitragen.

Allgemein muß man von einem Zusammenspiel von unmittelbaren Ausdrucks- und kontrollierten Darstellungsanteilen im nonverbalen Verhalten ausgehen.

\section{Multifunktionalität}

Für die verschiedenen Verhaltensweisen lassen sich eine Reihe von spezifischen Funktionen angeben. Für die Mimik ist es z. B. vor allem der Ausdruck von Affekten; in der Abfolge von Blickzuwendung und -abwendung finden sich Verbindungen zu momentanen kognitiven Vorgängen, zur Aufmerksamkeitsstruktur usw. (s. EKMAN u. FriesEN 1969; KendON 1967; PloOG 1980c). Auf diese spezifischen Funktionen soll hier nicht weiter eingegangen werden.

$\mathrm{Zu}$ bedenken ist, daß ein Verhalten nicht aus einer einzelnen Funktion heraus zu verstehen ist, sondern daß es gleichzeitig verschiedene Funktionen haben kann. So steuert die Blickzuwendung und -abwendung nicht nur die Art und Menge der eingehenden op- 
tischen Informationen, sie beeinflußt gleichzeitig auch den Gesprächsablauf als Signal beim Sprecherwechsel (ELLGRING 1981). Sie vermittelt weiterhin für den Partner den Eindruck wechselnder Aufnahmebereitschaft und damit emotionaler Beteiligung. Aus dem ,leeren Starren“ gewinnt man andererseits den Eindruck fehlender affektiver und kognitiver Beteiligung. Dieser Eindruck beruht im wesentlichen darauf, daß die ansonsten mit dem eigenen Sprechablauf und mit dem Partnerverhalten koordinierten Blickwendungen fehlen.

Zu trennen sind die Funktionen des Verhaltens für den Sender von denen für den Empfänger. Zwar werden sich Ausdruck und Eindruck im Normalfall wahrscheinlich entsprechen. Am Beispiel einer Fazialisparese wird allerdings deutlich, daß gerade im pathologischen Bereich eine starke Diskrepanz eintreten kann: Ein nicht mehr steuerbarer Ausdruck führt auf der Empfängerseite zu einem gestörten Eindrucksbild. Dieser Eindruck steuert wiederum das Verhalten des Empfängers, indem er z. B. das Gesicht des anderen weniger anblickt. Aus der Multifunktionalität folgt, daß man im Normalbereich keine einfachen oder isolierten Beziehungen zwischen psychischen Vorgängen und dem Ausdrucksverhalten erwarten kann. Im pathologischen Bereich wiederum sind die Eindrucksprozesse ebenso zu berücksichtigen wie die gestörten Verhaltenssteuerungen auf der Senderseite.

\section{Soziale Wirksamkeit}

Nonverbales Verhalten ist nicht nur ein Indikator oder Ausdruck psychischer Prozesse, sondern es beeinflußt in der sozialen Interaktion gleichzeitig das Verhalten und das Erleben anderer Personen. Diese doppelte Funktion des Verhaltens als Ausdruck von Antriebszuständen etc. und als Signal für andere Personen (s. PLOOG 1980 a) gewinnt besondere Bedeutung, wenn in diesem Bereich Störungen auftreten.

Eine Affektstörung ist dann nicht mehr ein individuelles Problem, sondern löst soziale Reaktionen aus, die wahrscheinlich zusätzliche Belastungen darstellen. Die „Affektinkontinenz" ist nicht nur als mangelnde Kontrollfähigkeit wie eine Harninkontinenz ein Problem für das Individuum. Auch die soziale Umgebung nimmt an der Störung teil, ist vielleicht sogar der Auslöser für den Affektausdruck. Wesensveränderungen bei Psychosen nimmt die soziale Umgebung zunächst am veränderten kommunikativen Verhalten wahr. Auf der anderen Seite wird die Unfähigkeit des Hemiplegikers, einen affektadäquaten Ausdruck zu zeigen, vor allem durch die soziale Bedeutung des Verhaltens, d.h. die vermutlichen oder tatsächlichen Reaktionen der anderen zu einem stark traumatischen Erleben.

Ausdrucksverhalten und seine Störungen sind also gleichzeitig sowohl im Hinblick auf das Individuum als auch auf seine soziale Wirksamkeit hin zu betrachten. Die soziale Wirksamkeit, die sich in den Reaktionen anderer manifestiert, kann z. B. eine Störung zusätzlich verstärken.

\section{Klinische Bedeutung}

Klinische Bedeutung gewinnt sozialkommunikatives Verhalten sowohl als Informationsquelle für die Diagnostik als auch für die Beurteilung von Veränderungen im Ver- 
lauf. Dabei sind es nicht nur die rein psychomotorischen oder kognitiven Funktionen, für deren Leistung bereits seit längerem standardisierte Testverfahren vorliegen, sondern vor allem auch affektive, appetitive und kurzfristige kognitive Prozesse, für die das nonverbale Verhalten als Informationsquelle dienen kann. Im klinischen Bereich beschränkte man sich bisher vor allem auf eindrucksmäßige Globalbeurteilungen, doch wurden in jüngerer Zeit auch Meßmethoden entwickelt, die Quantifizierungen dieser Verhaltensaspekte erlauben.

Außer der Möglichkeit, Informationen zu gewinnen, ist sozialkommunikatives Verhalten darüberhinaus ein Teil der sozialen Fertigkeiten, die wie die Sprache für den Umgang mit anderen beherrscht werden müssen. Insofern sind Defizite in diesem Bereich auch daraufhin zu betrachten, inwieweit sie durch systematisches Training behoben werden können. In dem vorliegenden Beitrag richtet sich das Augenmerk vor allem auf den diagnostisch-indikativen Wert des nonverbalen Verhaltens. Nicht notwendigerweise sind es „Leistungen“, die dabei ermittelt werden, sondern Informationen über ein affektives Geschehen, das sich sprachlich oft nur unzulänglich ausdrücken läßt.

\section{Affektausdruck bei hirnorganischen Veränderungen}

Vor allem in klinischen Beschreibungen finden sich Hinweise auf Veränderungen, Störungen und Ausfälle von kommunikativen Verhaltensweisen bei verschiedensten hirnorganischen Störungen (Bente u. Wieser 1953; Gerstenbrand 1967; Poeck 1969; WILLiAMS 1969; KolB u. MiLnER 1981).

Am deutlichsten wurden bisher bei Aphasikern Defizite in der Gestik, aber auch in der Pantomimik und in Bewegungsnachahmungen nachgewiesen (GooDGLASS u. KAPLAN 1963; GainotTI u. LEMmo 1976; VARNEY 1978). Sprachstörungen sind hier deutlich mit spezifischen motorischen Defiziten assoziiert. Ein wesentlicher Bestandteil hirnorganischer Psychosyndrome sind Veränderungen der Affektivität und des damit verbundenen mimischen Ausdrucks.

Einige allgemeine Informationen über die Steuerung der Mimik sollen hier vorangeschickt werden.

Die Gesichtsmuskulatur ist beim Menschen hochdifferenziert ausgebildet und erlaubt eine Vielzahl von Bewegungen, die auf der Hautoberfläche als mimische Erscheinungen sichtbar werden.

Die Gesichtsmuskeln werden durch einen einzigen, außerordentlich verästelten Hirnnerven, den N. facialis versorgt. An der Produktion der vielfältigen Innervationsmuster, die vom N. facialis ausgeführt werden, ist das motorische System von der Hirnrinde bis zu Mittelhirn und Brücke, aber auch das Limbische System beteiligt. Dabei besteht eine rechtshemisphärische Dominanz für den Ausdruck von Emotionen, die in einer stärkeren linksseitigen Expressivität der Mimik resultiert (WOLFF 1943; CAMPBELL 1978; Sackeim u. Gur 1978; SaCkeim et al. 1978; Borod u. Caron 1980). Gleichzeitig scheint eine Präferenz der rechten Hemisphäre für die Verarbeitung mimischer Informationen und beim Erkennen von Gesichtern zu bestehen (BRUYER 1980; LEEHEY et al. 1978; Rapaczynski u. Ehrlichman 1979; Young u. Bion 1980; Sergent u. Bindra 1981).

In der Regel bestehen diese Präferenzen für eine rechtshirnige Verarbeitung auch bei hirnorganischen Patienten (BRUYER 1979; CiCONE et al. 1980; Goldblum 1980). Aller- 
dings zeigen diese Patienten schlechtere Ergebnisse als Kontrollpersonen, wenn sie schematisierte Gesichter nach ihrer Ähnlichkeit vergleichen sollen, wobei wiederum bei rechtsseitigen Läsionen größere Schwierigkeiten bestehen (BRUYER U. VELGE 1980). Möglicherweise spielt hierbei eine Rolle, daß solche Patienten Schwierigkeiten mit sequentiellen Strategien bei der Informationsverarbeitung haben. Selbst bei einer einfachen Zuordnungsaufgabe, bei der gezeichnete und fotografierte Gesichter als fröhlich, neutral oder ärgerlich beurteilt werden sollten, zeigten Patienten mit chronischem hirnorganischem Syndrom deutlich schlechtere Leistungen als Patienten mit chronifizierter Schizophrenie oder affektiven Psychosen, die ihrerseits wie die Kontrollpersonen nahezu 100\%ig richtige Zuordnungen trafen (KuRUCZ et al. 1979). Beide Patientengruppen dieser Untersuchung hatten ein durchschnittliches Alter von etwa 70 Jahren und waren langjährig chronifiziert. Die Autoren schlagen daher korrespondierend zu BoDAMERS Prosopagnosie den Begriff der „prosopo-affektiven Agnosie“ für diese mangelnde Differenzierungsleistung bei hirnorganischen Patienten vor.

Im Gegensatz zu der vergleichsweise häufig untersuchten Erkennung von Gesichtern und Emotionen findet man allerdings vergleichsweise wenige systematische Untersuchungen über den mimischen Ausdruck bei hirnorganischen Patienten selbst. Dies ist erstaunlich, denn immer dann, wenn von Affekten im Zusammenhang mit hirnorganischen Veränderungen die Rede ist, sind wahrscheinlich mimische Ausdruckserscheinungen mit beteiligt. Zumindest geht mimisches Verhalten, wenn auch häufig implizit, in Beurteilungen und Beschreibungen von Affektauffälligkeiten wie Affektlabilität, Desintegration der Affekte, Affektstarre etc. mit ein.

Ähnlich wie bei defektschizophrenen Patienten zeigen sich bei hirnorganischen Patienten motorische Stereotypien in der Mimik, die in der mimischen Desintegration Primitivkoordinationen hervortreten lassen (Ploog 1964, S. 352 ff.; HeImanN u. SPOERRI 1957). In der Entdifferenzierung der auslösbaren Reize und in den schablonenhaften Reaktionen manifestieren sich elementare Störungen von Funktionen, die in die fundamentalen angeborenen Mechanismen hineinwirken.

Betrachten Patienten verschiedene emotional getönte Dias, so läßt sich der dabei gezeigte mimische Ausdruck von Patienten mit rechtsseitigen Hirnläsionen bzw. mit Parkinson-Erkrankung weniger eindeutig beurteilen als der von Patienten mit linksseitiger Läsion (Aphasiker). Zwischen Aphasikern (linksseitiger Läsion) und gesunden Kontrollpersonen bestand in dieser Hinsicht kein Unterschied. BRUYER (1981) forderte Patienten mit unilateraler Läsion (von denen die Hälfte jeweils eine kontralaterale Fazialisparese hatte) auf, einen neutralen, traurigen bzw. lächelnden Gesichtsausdruck darzustellen. Hier ergeben sich ähnliche Tendenzen: Bei linksseitiger Läsion erscheint die linke Gesichtshälfte, wie auch bei Kontrollpersonen in anderen Untersuchungen verschiedentlich gefunden, expressiver. Bei rechtsseitiger Läsion ist es, allerdings nur bei Patienten mit kontralateraler Parese, die rechte Gesichtshälfte. Die unterschiedliche Expressivität wird bei „,neutralem“ und „traurigem“ Gesicht, nicht aber beim Lächeln wahrgenommen.

Außer diesen wenigen, eher indirekten Hinweisen geben vor allem die klinischen Beschreibungen ein plastisches Bild von den Veränderungen des Affekts und der Mimik bei hirnorganischen Störungen. Von den psychischen Funktionen, die beim hirnorganischen Psychosyndrom deutlich verändert sind und somit auch einen wesentlichen Teil der Erkrankung ausmachen, ist der Affekthaushalt in ähnlichem Ausmaß betroffen wie die kognitiven Funktionen, die psychomotorischen Leistungen etc. So wird als zentrales 
Symptom die „Affektinkontinenz“ beschrieben, d.h. eine auffällige Labilität in der $\ddot{A u}$ Berung von Gefühlen (Tölle 1982, S. 268). Der Ausdruck der Gefühle steht dann in keinem Verhältnis zur Stimmung. Gleichzeitig verarmt der Affektausdruck und verliert seine Differenziertheit und Spontaneität (BLEULER 1969, S. 184).

Abgesehen von solchen Hinweisen bei diffusen hirnorganischen Störungen finden sich Beobachtungen, die die Verhaltensänderungen präzise auf hirnlokale Störungen beziehen. Das pathologische Lachen und Weinen ist ein markantes Beispiel (WILSON 1924; PoEck 1969, S. 356 ff.). Diese mimischen Reaktionen erfolgen unkontrollierbar, in Reaktion auf unspezifische Stimuli, ohne den dazugehörigen Affekt. Nach PoECK (1969, S. 362) können Läsionen auf verschiedenen Ebenen pathologisches Lachen und Weinen bedingen: Läsionen der basalen Ganglien, der Substantia nigra, des Hypothalamus, bilaterale Läsionen des Pyramidaltrakts und möglicherweise auch thalamischer Gebiete.

Auf eine Enthemmung der Mimik bei Ausfällen in der Pyramidenbahn weist ZüLCH (1951, S.405) hin, wobei auf der Lähmungsseite bei Hemiplegie der Ausdruck hypermimisch ausfallen kann.

Sehr detaillierte Beschreibungen des Ausdrucksverhaltens bei traumatischem apallischem Syndrom gibt Gerstenbrand (1967, S. 54 ff.), wobei er besonders auch die Reintegration der Affektivität behandelt.

Im Vollstadium des apallischen Syndroms fehlen emotionale Reaktionen, während im Defektstadium eine emotionelle und affektive Enthemmung beschrieben wird. Mit Besserung tritt eine Differenzierung der Mimik ein, die zunächst ängstliche Aspekte aufweist und sich auch hierin weiter differenziert. Später kommen Entspannungsreaktionen und auch mimische Unmutsreaktionen hinzu. Die differenzierten Reaktionen von Freude und Trauer treten nach GERSTENBRAND relativ spät in Erscheinung. Sie sind dann vor allem an das Wiedererkennen von Personen gebunden.

Beim traumatischen apallischen Syndrom finden sich also deutliche Hinweise darauf, daß die Mimik differenziert über die Reorganisation des Affektsystems Auskunft geben kann. Bedeutsam erscheint hier, daß positiver Affektausdruck erst zu einem relativ späten Zeitpunkt des Wiederherstellungsprozesses auftritt.

Eine Erkrankung, deren Symptomatik vor allem bei älteren Patienten Ähnlichkeiten zum hirnorganischen Psychosyndrom aufweisen kann, ist das Parkinson-Syndrom. Für unser Thema ist diese Erkrankung insofern wichtig, als bei dieser Störung des extrapyramidalen Systems, einer nigrostriären Degeneration im dopaminergen System (s. BIRKMAYER u. RIEDERER 1980) deutliche Veränderungen der Ausdrucksmotorik in Mimik, Gestik, Haltung und Gang auftreten: Die Mimik ist maskenhaft starr und steif, wobei auch ein starres Lächeln auftreten kann; in der Motorik sind Tremor und Rigor zentrale Hinweise (SELBY 1968). Dem maskenhaften Gesicht entspricht allerdings keineswegs ein fehlendes affektives Erleben. Es reflektiert lediglich die Unfähigkeit, das affektive Erleben in mimische Aktivität umzusetzen. Nach BIRKMAYER u. RIEDERER (1980, S.68) kann sich diese Amimie nach einer Dopa-Injektion vollständig auflösen, und es entsteht ein ausdrucksvolles, lebhaftes Gesicht.

Auf der einen Seite ist es also klinisch evident, daß a) Emotionalität und Mimik eng miteinander verknüpft sind, b) beides als Bestandteil hirnorganischer Störungen verändert ist und c) das Ausdrucksverhalten wichtige Hinweise für die Erkennung hirnorganischer Erkrankungen und ihrer Veränderungen liefert. Auf der anderen Seite fehlen allerdings weitgehend Untersuchungen, die das veränderte Verhalten systematisch erfassen. Diese Diskrepanz kann verschiedene Gründe haben. 
Es ist unwahrscheinlich, daß systematische Untersuchungen des Ausdrucks als Teil des psychischen Geschehens den notwendigen Aufwand nicht lohnen. Reduktion oder Dissoziation von Verhalten und Erleben werden jedenfalls vom Kliniker als wichtige diagnostische Hinweise verwendet.

Das Phänomen könnte wiederum so evident sein, daß systematische Untersuchungen überflüssig wären. Dieser Grund würde allerdings auch für andere Phänomene gelten, wie Veränderungen von psychomotorischen oder kognitiven Leistungen, die trotz ihrer Evidenz extensiv untersucht wurden.

Es scheint vielmehr vor allem an den methodischen Schwierigkeiten zu liegen, daß bei einem prägnanten, klinisch bedeutsamen Phänomen systematische Untersuchungen bisher kaum zu finden sind. Die mimischen Verhaltensweisen selber sind z. B. häufig sehr kurz, dauern nach eigenen Beobachtungen meist weniger als zwei Sekunden. Sie müssen also im Zeitlupentempo beobachtet werden. Dies wurde ökonomisch erst durch Video möglich. Mimische Aktionen gehen zudem auf komplexe Muskelaktivationen zurück, lassen sich also nur schwer in einzelnen Dimensionen beschreiben. Beobachtung, Beschreibung und Interpretation sind meist eng miteinander verquickt. Man nimmt ein freundliches, aufgesetztes, falsches, offenes oder gequältes Lächeln wahr, wobei die Verhaltenselemente im Ausdruck, die zu diesem Eindruck führten, kaum bewußt analysiert werden.

Die Frage ist daher, in welcher Weise trotz dieser Schwierigkeiten ein methodisch akzeptabler Zugang zum nonverbalen Ausdrucksverhalten gefunden werden kann.

\section{Veränderungen des nonverbalen Ausdrucksverhaltens bei affektiven Störungen}

Am Beispiel der Depression soll solch ein methodischer Weg für die Analyse des Ausdrucksverhaltens im Verlauf von eindeutig affektiven Störungen aufgezeigt werden. Über den methodischen Gesichtspunkt hinaus ist die Depression zudem im Zusammenhang mit hirnorganischen Störungen interessant. Auch dort treten depressive Symptome auf, und es ergeben sich durchaus differentialdiagnostische Probleme, vor allem bei Beginn von organischen Psychosen (s. BLEULER 1969, S. 185).

Vergleichbar zum Stand der Forschung bei hirnorganischen Störungen finden sich zahlreiche klinische Beobachtungen und Beschreibungen, aber nur wenige systematische Untersuchungen des nonverbalen Verhaltens bei depressiven Patienten. In diesen Untersuchungen wiederum zeigten sich, verglichen mit dem deutlichen klinischen Eindruck, nur erstaunlich geringe Unterschiede zwischen depressiven und anderen psychiatrischen Patienten bzw. Kontrollpersonen, sofern man statistische Gruppenvergleiche heranzieht (RutTer u. StePhenson 1972; Jones u. PANSA 1979; Wallbott 1982). In einer Untersuchung von FiscH et al. (1983) an 13 depressiven Patienten zeigte sich in dem von ihnen erfaßten motorischen Verhalten (Gestik, Körperhaltung etc.) für den Aspekt der Motilität eine hohe interindividuelle Variation, die keinen bedeutsamen Zusammenhang mit dem Befinden ergab. Der aus den Daten ebenfalls abgeleitete Kennwert motorischer Komplexität, d.h. die Vielfalt eingenommener Positionen von Körper- und Kopfhaltung, Gestik etc. korrelierte hingegen hoch mit dem klinischen Besserungsurteil. 
Auch wenn in den verschiedenen Untersuchungen statistisch signifikante Gruppenunterschiede gefunden wurden, fallen sie insgesamt keineswegs so deutlich aus, wie man sie nach klinischen Beschreibungen erwarten würde.

Im folgenden sollen einige Ergebnisse aus einer eigenen Untersuchung ${ }^{1}$ berichtet werden, in der nicht Gruppen verglichen, sondern vor allem der Verlauf von Verhalten und subjektivem Befinden intraindividuell aufeinander bezogen wurde. Hiermit sollte festgestellt werden, wie sich Änderungen des subjektiven Befindens, d.h. der allgemeinen Affektlage, im nonverbalen Verhalten manifestieren. Dabei war zu erwarten, daß sich bei Besserung des depressiven Zustands die Verhaltensreduktion auflöst, daß also aktiv kommunikatives Verhalten zunimmt und sich das Repertoire erweitert.

Zudem ergab sich die Frage, wie sich Zusammenhänge von Verhalten und Erleben manifestieren, die zwar im klinischen Eindruck evident erscheinen, die sich allerdings bei Verhaltensmessungen kaum so deutlich wiederfinden lassen. Übertreibt also der klinische Eindruck einen nur mäßig nachweisbaren Zusammenhang?

\section{Methodik}

Untersucht wurden 20 endogen, 16 neurotisch depressive Patienten und 9 Kontrollpersonen über den Verlauf des Klinikaufenthalts hinweg. Während ihres stationären Aufenthalts, der auch für die Kontrollpersonen von den äußeren Bedingungen her vergleichbar war, wurden klinische Interviews video-aufgezeichnet. Diese Interviews bestanden aus einem standardisierten Teil und einem freien Gespräch. Ausgewertet wurden jeweils 5 Minuten dieser Interviews.

Als Maße des nonverbalen Verhaltens wurden die relative Zeitdauer von Blickzuwendung und Sprechaktivität, die Anzahl sprachbegleitender Gesten bezogen auf die Sprechmenge verwendet und zudem verschiedene Parameter, die sich aus der Mimikanalyse ergaben. Die Mimikanalyse enthält drei Bestandteile: 1. Beschreibung der mimischen Aktionen, 2. Bestimmung der Parameter von allgemeiner und spezifischer mimischer Aktivität bzw. des Repertoires, 3. inhaltliche Bestimmung von Mustern mimischer Aktivität.

Die Beschreibung des mimischen Verhaltens folgte nach dem Facial Action Coding System (FACS) von EKMAN u. FRIESEN (1978), das wiederum auf ein Verfahren des dänischen Anatomen HJorTsJoe (1970) zurückgeht. Darin werden Action Units (AU) einzeln oder in Kombination nach bestimmten Kodierungsregeln notiert. Im Stirnbereich wird beispielsweise das Hochziehen der äußeren Augenbrauen mit AU 2, das der inneren Augenbrauen mit AU 1 und das Zusammenziehen der Brauen mit AU 4 gekennzeichnet. Diese einzelnen AUs sind wiederum kombinierbar. Wenn z. B. gleichzeitig die inneren Augenbrauen angehoben und zusammengezogen werden, so wird die AU $1+4$ codiert.

Insgesamt läßt sich so das mimische Verhalten in Elementen bzw. in Kombination beschreiben, wobei sichtbare Veränderungen der Gesichtsoberfläche auf einer funktional-anatomischen Basis codiert werden.

Jeweils 2 bis 3 Interviews von Patienten und Kontrollpersonen, insgesamt 120 Inter-

${ }^{1}$ Die Untersuchung war auch Teil eines interdisziplinären Projektes am Max-Planck-Institut für Psychiatrie über zirkadiane Periodik in der Depression. 
views, wurden auf diese Weise codiert. Mit wiederholten Messungen bei den Kontrollpersonen konnte die Stabilität des Verhaltens bei vergleichsweise homogenem emotionalem Befinden festgestellt werden. Bei den Patienten wurden Interviews vom Beginn und Ende des Klinikaufenthalts und aus einer Nachuntersuchung herangezogen. Angaben über das subjektive Befinden lieferte eine visuelle Analogskala (,Zustandsbarometer“, FolSTEIN u. LuRIA 1973). Auf verschiedenen anderen Skalen wurden zudem der klinische Eindruck global (SCHWARZ u. STRIan 1972) und nach Merkmalsbereichen differenziert erfaßt. Einzelheiten dazu sind in einem standardisierten Interview zur Verlaufsuntersuchung depressiver Erkrankungen (SID, ELLGRING et al. 1978) festgelegt.

\section{Ergebnisse}

Bei den Patienten treten im Zustand der Depression verglichen mit den Kontrollpersonen deutlich reduzierte Werte in den verschiedenen Merkmalen des nonverbalen Verhaltens auf; sie nehmen bei Besserung des Befindens in vielen Fällen wieder substantiell
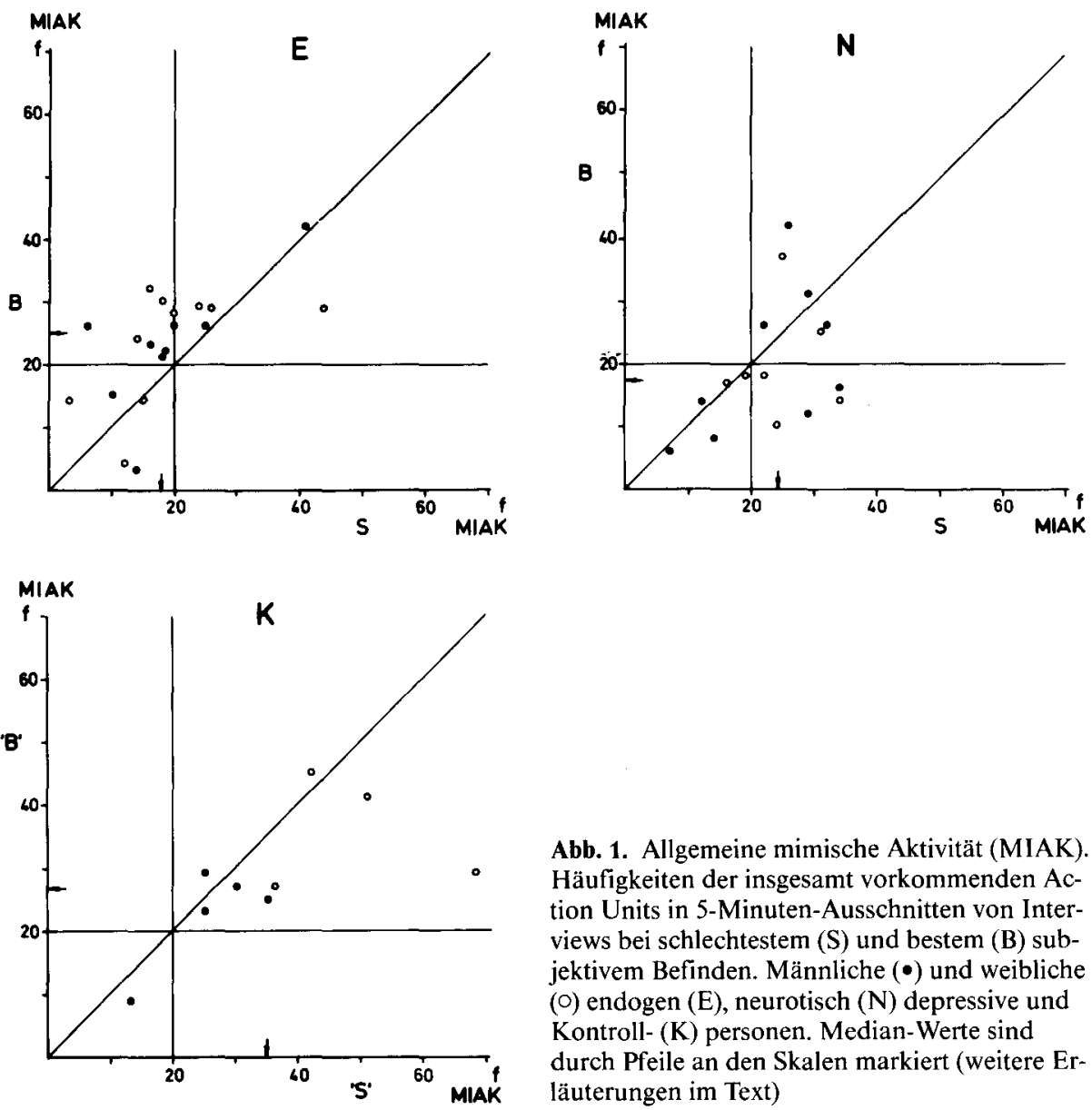

Abb. 1. Allgemeine mimische Aktivität (MIAK). Häufigkeiten der insgesamt vorkommenden Action Units in 5-Minuten-Ausschnitten von Interviews bei schlechtestem (S) und bestem (B) subjektivem Befinden. Männliche $(\bullet)$ und weibliche $(O)$ endogen $(E)$, neurotisch $(N)$ depressive und Kontroll- $(\mathrm{K})$ personen. Median-Werte sind durch Pfeile an den Skalen markiert (weitere Erläuterungen im Text) 
zu. Allerdings, und dies ist besonders hervorzuheben, gelten diese Veränderungen individuenspezifisch.

Am Beispiel der allgemeinen mimischen Aktivität, d.h. der Anzahl vorkommender mimischer Aktionen pro $5 \mathrm{~min}$, soll das Ergebnis exemplarisch erläutert werden.

Bei einem erheblichen Teil, vor allem der endogen depressiven Patienten, ist die allgemeine mimische Aktivität gegenüber der der Kontrollpersonen reduziert. Bei besserem Befinden nimmt die mimische Aktivität wieder zu. Ein Teil der neurotisch depressiven Patienten zeigt allerdings bei besserem Befinden weniger mimische Äußerungen und damit ein erwartungskonträres Verhalten.

Die Abb. 1 stellt in ihren drei Teilen die individuellen Werte mimischer Aktivität bei schlechtestem und bestem Befinden dar.

In der Darstellung markieren die Kennlinien bei 20, d.h. 4 AU's pro Minute, die Grenze reduzierter Werte, die sich aus den intraindividuell konstanten und allgemein höheren Werten der Kontrollpersonen ergibt.

Die Diagonale trennt die positiven bzw. negativen Veränderungen. Punkte oberhalb der Diagonale zeigen an, daß die mimische Aktivität bei besserem Befinden gegenüber der bei schlechterem Befinden zunimmt. Punkte unterhalb der Diagonalen, daß sie bei Besserung abnimmt. Zieht man auch die Werte bei „mittlerem" Befinden heran, die in Abb. 1 nicht enthalten sind, so ergibt sich eine deutlich gegenläufige Tendenz: Bei den endogen depressiven Patienten geht der Anteil reduzierter Werte signifikant von 75\% über $43 \%$ auf $25 \%$ zurück, während er bei den neurotisch depressiven Patienten signifikant von 31\% über 38\% auf 63\% zunimmt (Fischer-Exakt-Tests: $\mathrm{p}<0,05$ ).

Bei schlechtem wie bei besserem Befinden liegen die Werte der Patienten signifikant unter denen der Kontrollpersonen (U-Test: $p<0,01$ ).

Intraindividuell nimmt bei 11 der 36 Patienten $(=31 \%)$ die mimische Aktivität bei Besserung des Zustands substantiell, d.h. hier um mehr als ein Drittel des Ausgangswertes zu.

Die Abb. 2 stellt die Veränderungsquotienten für jede Person dar. In den Veränderungsquotienten wird die Differenz zwischen dem Wert bei bestem bzw. mittlerem $\mathrm{Zu}$ stand einerseits und dem bei schlechtestem Zustand andererseits auf den Ausgangswert bei schlechtestem Zustand bezogen.

Auch hier zeigt sich eine gegenläufige Tendenz: Bei einem größeren Teil der endogen depressiven Patienten nimmt die mimische Aktivität substantiell, d.h. um mehr als 33\% des Ausgangswertes zu. Immerhin 5 der $16(=31 \%)$ neurotisch depressiven Patienten vermindern substantiell ihre mimische Aktivität bei besserem Befinden.

Für das Lächeln (AU 6+12) als eine spezifische mimische Aktivität, die im Gespräch häufig als Signal, ähnlich wie das Kopfnicken, auftreten kann oder aber wechselseitig von den Gesprächspartnern ausgelöst wird, gilt ähnliches: Auch dieses Verhalten nimmt, allerdings wiederum individuenspezifisch, aus reduzierten Werten heraus substantiell zu. 28 der 36 Patienten $(=78 \%$ ) zeigen bei schlechtem Befinden reduzierte Werte, bei $22(=61 \%)$ erhöht sich die Häufigkeit des Lächelns bei Zustandsbesserung deutlich nach den gesetzten Kriterien.

Zwei Punkte sind anzumerken: Auch bei Besserung des Befindens kann, besonders bei neurotisch depressiven Patienten $(=17 \%)$, die Häufigkeit der Lächelreaktion substantiell abnehmen. Weiterhin sind Veränderungen der allgemeinen mimischen Aktivität nicht notwendig an solche der spezifischen mimischen Aktivität in bestimmten Gesichtsbereichen gekoppelt. 


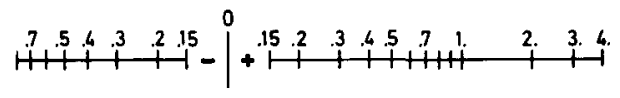

E

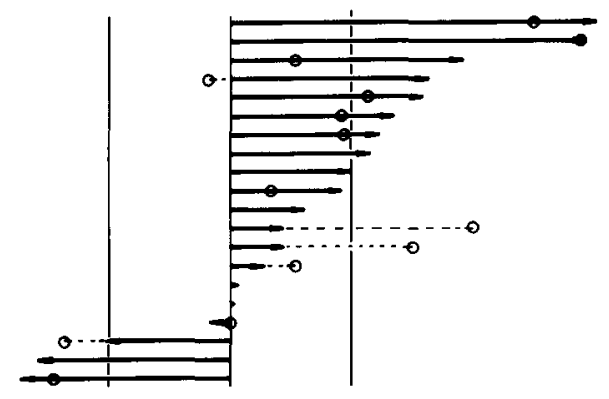

Abb. 2. Veränderungsquotienten der allgemeinen mimischen Aktivität (MIAK). Zunahme bzw. Abnahme bezogen auf den Ausgangswert bei endogen (E), neurotisch (N) depressiven und Kontroll- (K) Personen. $\rightarrow=$ Werte des Extremvergleichs zwischen bestem (B) und schlechtestem (S) subjektiven Befinden ((B-S)/S), o-Werte des Vergleichs zwischen mittlerem (M) und schlechtestem (S) subjektiven Befinden $((\mathrm{M}-\mathrm{S}) / \mathrm{S})$. Die Kennlinie bei $\mathrm{VO}=33$ gibt die kritische Grenze für substantielle Veränderungen an. Semilogarithmische Darstellung mit linearen Werten zwischen 0 und 0,1 . Jede Person ist in einer Linie repräsentiert

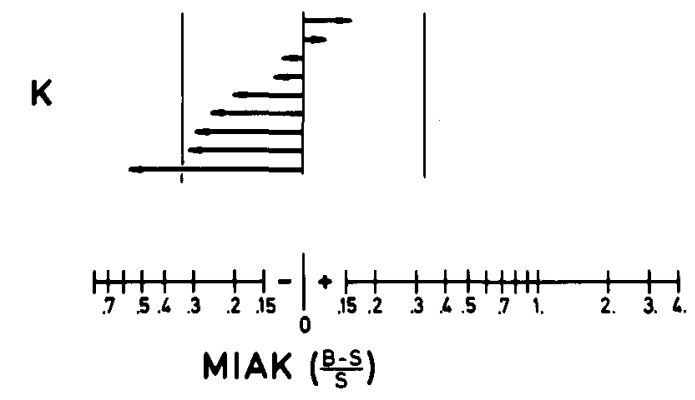

Das Repertoire ist vergleichsweise selten reduziert (25\%) und nimmt auch seltener (bei 43\% der Patienten) substantiell bei Zustandsbesserung zu.

Für die inhaltliche Kennzeichnung der mimischen Aktivität wurden in einem weiteren Schritt mit Hilfe eines Clusterverfahrens (Ward-Methode, STEINHAUSEN u. LANGER 1977) Muster ähnlicher Konfigurationen nach den relativen Häufigkeiten bestimmt, mit denen einzelne mimische Elemente bei einer Person in einer Situation auftraten. Es ergaben sich 5 Cluster, in die sich sämtliche analysierten Interviews von Patienten und Kontrollpersonen einordnen ließen (s. Abb.3).

Es zeigt sich, daß in einigen Clustern nur depressive Patienten anzutreffen sind, daß weiterhin bei Änderung des Befindens Personen anderen Gruppierungen zugeordnet 

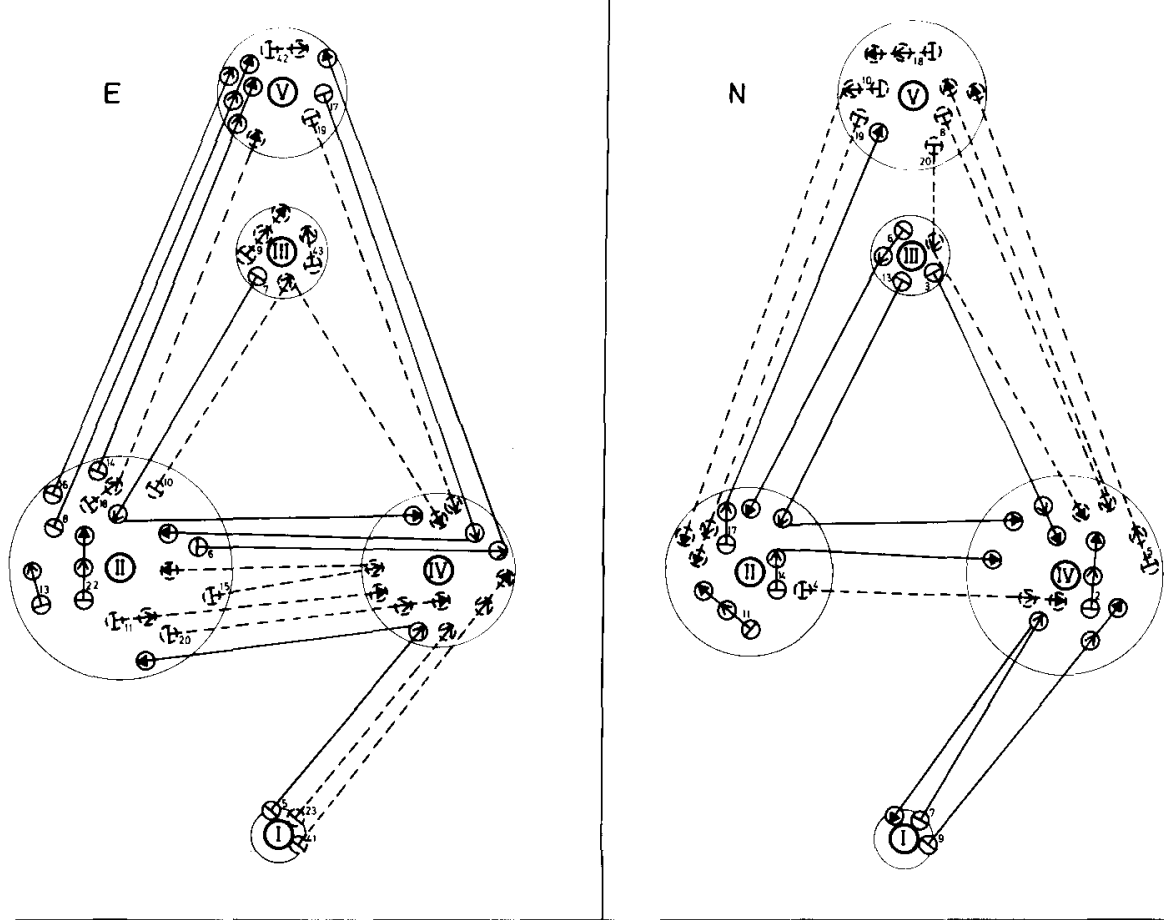

K

(V)

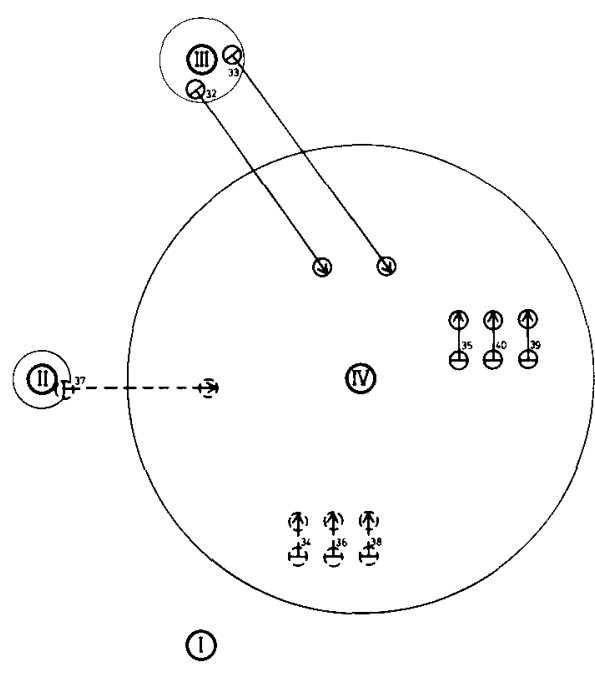

Abb. 3. Cluster der Muster mimischer Aktivität. Die Größe der Kreise entspricht dem Anteil des jeweiligen Clusters in der Gruppe der endogen $(\mathrm{E})$, neurotisch $(\mathrm{N})$ depressiven und Kontroll- (K) Personen. Für jede Person gibt die Pfeilrichtung die Veränderung vom Anfangszum End- und Nachkontrollinterview an. $\oplus$ Anfangs- $\Theta$ End- $\oplus$ Nachkontroll-Interview $\cdots$ o 

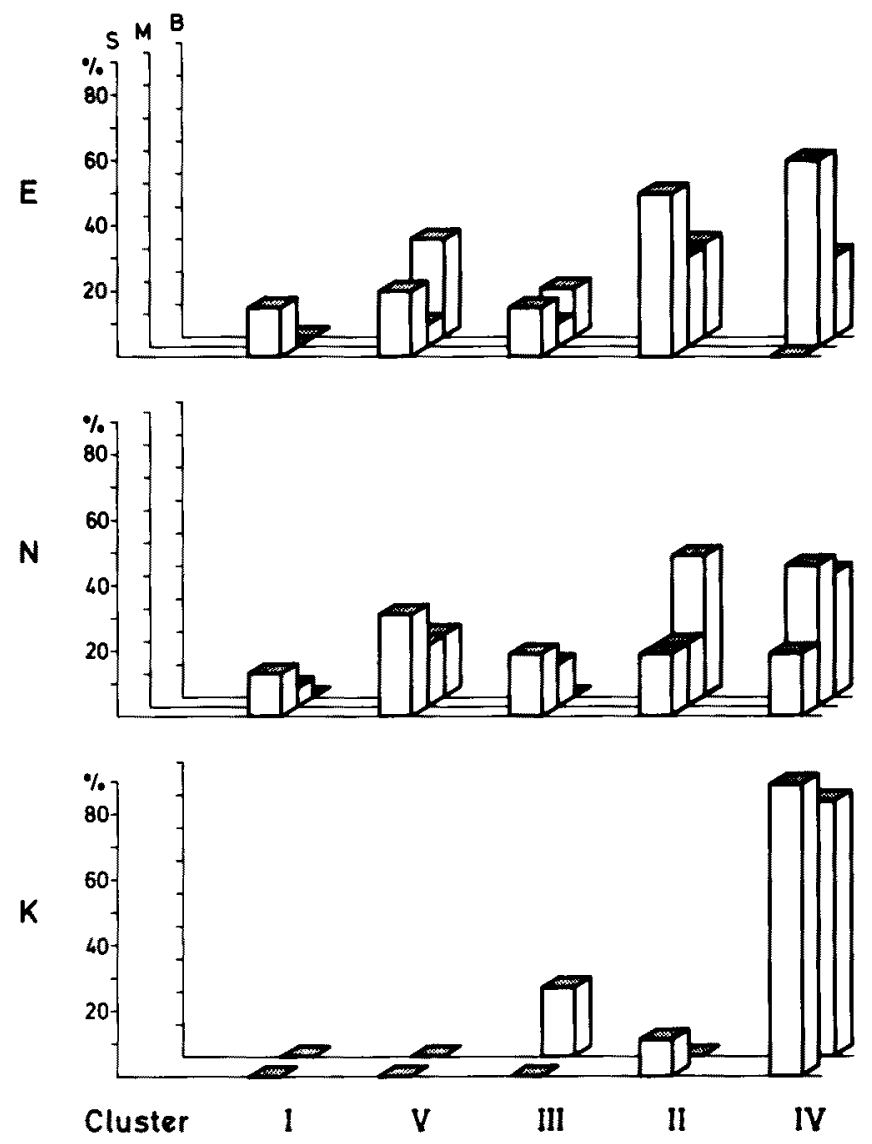

Abb. 4. Anteil der Cluster bei unterschiedlichem subjektiven Befinden von endogen (E), neurotisch (N) depressiven und Kontroll- (K) Personen. Die Höhe der Säulen gibt den Anteil (\%) der Personen an, die zu dem jeweiligen Zustand (schlechtestem $=S$, mittlerem $=M$, bestem $=B$ Befinden) in den verschiedenen Clustern (I, V, . I IV) repräsentiert

werden können. Die Kontrollpersonen ( 15 der 18 Kontrollpersonen $=83 \%$ ) zentrieren sich im Gegensatz zu der beobachteten Vielfalt der Patientenmuster im wesentlichen auf das Cluster IV. Die Abb. 4 stellt die Anteile der Cluster bei unterschiedlichem subjektivem Befinden zusammengefaßt dar.

Eindeutig ist der hohe Anteil des Clusters IV mit $89 \%$ bzw. $78 \%$ für die Kontrollpersonen. In diesem Cluster befinden sich keiner der endogen, allerdings $3(=19 \%)$ der neurotisch depressiven Patienten im schlechtesten Zustand. Cluster I und nicht so eindeutig auch Cluster V erscheinen spezifisch für die depressiven Patienten. Hier finden sich keine Kontrollpersonen und auch keine Interviews im besten Zustand. Gegenläufig verhalten sich die Anteile von Cluster II für endogen und neurotisch depressive Patienten. Im Gegensatz zu den endogenen findet man bei Besserung des Befindens in diesem Cluster häufiger neurotisch depressive Patienten.

Auf die inhaltliche Interpretation der Muster muß hier weitgehend verzichtet werden. Sie läßt sich aus den Zentroidwerten der einzelnen AU's in den verschiedenen Clu- 
Tabelle 1. Anzahl der Merkmale mit reduzierten und substantiell zunehmenden Werten. Anzahl (n) und Anteile (\%) der depressiven Patienten, die reduzierte bzw. substantiell zunehmende Werte in verschiedenen Merkmalen (m) nonverbalen Verhaltens zeigen. Zustände relativ schlechtesten (S), mittleren ( $M$ ) und besten (B) subjektiven Befindens

\begin{tabular}{|c|c|c|c|c|c|}
\hline \multirow{3}{*}{$\begin{array}{l}\text { Anz.d. } \\
\text { Merkmale } \\
\mathrm{m}\end{array}$} & \multicolumn{3}{|c|}{ Reduzierte Werte im Zustand } & \multicolumn{2}{|c|}{$\begin{array}{l}\text { Substantielle Zunahme } \\
\text { des Verhaltens }\end{array}$} \\
\hline & $S$ & $M$ & B & $S \rightarrow M$ & $\mathrm{~S} \rightarrow \mathrm{B}$ \\
\hline & $\overline{\mathrm{n}} \%$ & $\mathrm{n} \%$ & $\mathrm{n} \%$ & $\bar{n} \%$ & n $\%$ \\
\hline 6 & $1(3 \%)$ & $-\quad-$ & - & - & $1(3 \%)$ \\
\hline 5 & $4(11 \%)$ & $2(6 \%)$ & $1(3 \%)$ & $1(3 \%)$ & $3(8 \%)$ \\
\hline 4 & $4(11 \%)$ & $1(3 \%)$ & $2(6 \%)$ & $6(20 \%)$ & \\
\hline 3 & $6(17 \%)$ & $2(6 \%)$ & $9(25 \%)$ & $3(10 \%)$ & $8(22 \%)$ \\
\hline 2 & $11(31 \%)$ & $8(27 \%)$ & $6(17 \%)$ & $9(30 \%)$ & $12(33 \%)$ \\
\hline 1 & $7(19 \%)$ & $9(30 \%)$ & $8(22 \%)$ & $7(23 \%)$ & $9(25 \%)$ \\
\hline 0 & $3(8 \%)$ & $8(27 \%)$ & $10(28 \%)$ & $4(13 \%)$ & $3(8 \%)$ \\
\hline$\Sigma$ & 36 & 30 & 36 & 30 & 36 \\
\hline
\end{tabular}

stern ableiten, erfordert allerdings eine detaillierte Beschreibung von Verhaltenselementen und deren möglichem affektivem Bedeutungsgehalt. Bemerkenswert ist das Muster IV als normales „Konversationsmuster“. Die dominierenden Verhaltensweisen sind hier die als „Sprechersignale“ zu interpretierenden AU's mit gehäuftem Anheben der Augenbrauen und Lächeln. Bei den depressionspezifischen Mustern fällt auf, daß die dominierenden mimischen Verhaltensweisen als Indikatoren von Ärger, Angst und Abscheu interpretiert werden können, mimische Signale der traurig-gedrückten Stimmung hingegen selten auftreten. Man könnte daraus ableiten, daß sich in den aktiven mimischen Äußerungen Teilkomponenten eines normalerweise zusammengehörigen Gesamtausdrucks überlagern.

Nimmt man die anderen Merkmale nonverbalen Verhaltens, d.h. die relative Dauer von Blickzuwendung, Sprechaktivität und die Menge der sprachbegleitenden Gestik hinzu, so ergibt sich eine erhebliche Vielfalt nonverbaler Reaktionsmuster, in denen sich abhängig vom subjektiven Befinden Veränderungen zeigen. Bei den meisten Patienten sind dabei nur wenige der möglichen Parameter nonverbalen Verhaltens im depressiven Zustand reduziert bzw. nehmen bei Zustandsbesserung substantiell zu (s. Tabelle 1).

Bei der Hälfte der Patienten betrifft die Reduktion weniger als drei der sechs erfaßten Parameter. Bei Zustandsbesserung nehmen meist auch nur weniger als drei der Parameter substantiell zu.

Festzuhalten ist, daß im Vergleich zu Kontrollpersonen depressive Patienten auch bei Besserung immer noch erheblich reduzierte Werte vor allem in den hier definierten Parametern der Mimik zeigen. Die Zahl der bei den einzelnen Personen noch reduzierten Parametern geht jedoch deutlich zurück.

\section{Interkorrelationen}

In den Interkorrelationen zeigen die Verhaltensmerkmale und das subjektive Befinden höchstens mittlere generelle Zusammenhänge, obwohl im Einzelfall die Verbindungen teilweise sehr eng sind (s. Tabelle 2). 
Tabelle 2. Interkorrelationen. $\mathrm{MIAK}=$ Allgemeine mimische Aktivität, $\mathrm{AU} 6+12=$ Häufigkeit des Lächelns, REP $2=$ Mimisches Repertoire, B $\%=$ Relative Dauer der Blickzuwendung, $\mathrm{S} \%=$ Relative Dauer der Sprechaktivităt, GAR = Menge der Gestik bezogen auf 5 min Sprechdauer, $Z B=S u b-$ jektives Befinden im Zustandsbarometer, $\mathrm{KE}=\mathrm{Klinischer}$ Eindruck. $\mathrm{N}=90$ Interviews, jeweils 2 Werte von $\mathrm{N}=45$ Personen. Kritische Korrelation für $\mathrm{N}=45: \mathrm{r}=+/-0,30$ für $\mathrm{p}=0,05$ und $r=+/-0,40$ für $p=0,01$

\begin{tabular}{|c|c|c|c|c|c|c|c|c|}
\hline Merkmal & MIAK & AU $6+12$ & REP 2 & $\mathrm{~B} \%$ & $\mathrm{~S} \%$ & GAR & $\mathrm{ZB}$ & $\mathrm{KE}$ \\
\hline MIAK & - & 0,60 & 0,66 & 0,16 & 0,09 & 0,11 & 0,17 & 0,26 \\
\hline AU 6+ 12 & & - & 0,33 & 0,33 & 0,26 & 0,09 & 0,49 & 0,60 \\
\hline REP 2 & & & - & 0,12 & 0,06 & 0,12 & 0,08 & 0,17 \\
\hline $\mathrm{B} \%$ & & & & - & 0,18 & 0,21 & 0,52 & 0,40 \\
\hline $\mathrm{S} \%$ & & & & & - & 0,16 & 0,41 & 0,41 \\
\hline GAR & & & & & & - & 0,08 & 0,02 \\
\hline ZB & & & & & & & - & 0,86 \\
\hline $\mathrm{KE}$ & & & & & & & & - \\
\hline
\end{tabular}

Die am häufigsten und stärksten mit dem Befinden assoziierten Merkmale Blickzuwendung $(B \%)$ und Lächeln (AU 6+12) korrelieren mit $r=0,52$ und $r=0,49$ höchst signifikant mit den Werten des subjektiven Befindens, ähnlich wie die Sprechaktivität mit $\mathrm{r}=0,41$ (sämtlich $\mathrm{p}<0.01$ bei einem $\mathrm{N}$ von 45 ). Nahezu identisch sind die entsprechenden Korrelationen zum „,klinischen Eindruck“: r(B\%-KE)=0,40, r(AU 6+12-KE) $=0,60, \mathrm{r}(\mathrm{S} \%-\mathrm{KE})=0,41$.

Die allgemeine mimische Aktivität korreliert zwar signifikant, aber niedrig mit dem klinischen Eindruck $(r=0,26)$ und noch niedriger $(r=0.17)$ mit den Zustandswerten (ZB). Für die gestischen Merkmale gehen die entsprechenden Korrelationen nicht über $r=0,08$ hinaus.

Die Maße verschiedener Verhaltensmerkmale korrelieren abgesehen von den Mimikparametern zu höchstens $r=0,33$ miteinander. Die Korrelationen basieren auf jeweils zwei Werten aus dem relativ besten und schlechtesten Zustand einer Person.

Obwohl einige der Korrelationen die allgemein zu erwartenden Zusammenhänge von Verhaltens- und subjektiven Maßen übersteigen, so umschreiben sie dennoch, ausgedrückt in Determinationskoeffizienten, nur zwischen $17 \%$ und $30 \%$ gemeinsamer Varianz. Als allgemeine Kennzeichnung von Populationen geben sie hier die individuellen Assoziationen nur unvollkommen wieder.

\section{Resümee}

In der Depression zeigt sich, anders als zunächst erwartet, eine erstaunliche Vielfalt von Ausdrucksweisen, die von Individuum zu Individuum in unterschiedlicher Art und Weise mit dem veränderten subjektiven Befinden verbunden sind. Im Sinne logischer OderVerbindungen treten bei den Verhaltensmerkmalen in verschiedenen Kombinationen reduzierte Werte auf und verändern sich bei Zustandsbesserung substantiell. Nur in wenigen Fällen sind sämtliche Merkmale betroffen; meist sind es, ohne erkennbare Abhängigkeit untereinander, nur einige von ihnen. Wenige Patienten zeigen entgegen der Erwartung keinerlei Veränderungen oder gar substantielle Verhaltensreduktionen bei Zustandsbesserung. 
Die potentiell verfügbaren Indikatoren treten nur partiell und unabhängig voneinander als Ausdruck des depressiven Zustands in Erscheinung. Bei einer Person kann dies etwa im Lächeln und in der übrigen mimischen Aktivität der Fall sein, bei einer anderen in der Blickzuwendung und im Sprechen.

Diese zwei Elemente, die intraindividuelle Assoziation und die Oder-Verbindung der einzelnen nonverbalen Verhaltensweisen, erscheinen für den Ausdruck der Stimmung und des Befindens wichtig: Intraindividuell lassen sich Zusammenhänge nachweisen, die interindividuell dadurch wieder verwischt werden können, daß andere Personen in dem jeweils betrachteten Verhaltensaspekt keine zustandsabhängigen Veränderungen zeigen. Für das Individuum besteht eine Reaktionsspezifität dergestalt, daß sich nur ein Teil des verfügbaren Verhaltensrepertoires abhängig vom psychischen Zustand verändert.

Auf das Konomieprinzip, das hier für den Ausdruck erkennbar wird und auf die partneradaptativen Prozesse in der interpersonalen Kommunikation, die sich aus der individuenspezifischen Verwendung nonverbaler Signale ergeben, kann hier nur verwiesen werden.

Folgerungen für die klinische Verhaltensbeurteilung und die systematische Erfassung des Ausdrucksverhaltens

\section{Klinische Inferenz}

Der vergleichsweise homogene Zustand der Depression drückt sich in einer Vielzahl von Verhaltensweisen und Verhaltensmustern aus, die als Eindruck vom Beobachter wieder zu einem homogenen Bild zusammengefaßt werden. Wenn die Depression aus verschiedenen Faktoren, dispositioneller, sozialer, kognitiver, biochemischer, physiologischer etc. Art heraus in einem „final common pathway“ mündet (AKISKAL 1979; PloOG 1980b), so entfaltet sich dieser Zustand wieder in den nonverbalen Verhaltensweisen.

Geht man von den individuenspezifischen Reaktionsformen aus, so ergibt sich die Frage, wie dieses Verhalten als Information zu einem klinischen Urteil, speziell der Attribution eines Zustands, beitragen kann. Daß nonverbale Informationen hierzu im unmittelbaren intraindividuellen Vergleich sehr differenziert genutzt werden können, zeigen die Untersuchungen von RENFORDT U. BUSCH (1978), vor allem aber auch Arbeiten von Pattay (1982) und Avarello (1983). Sie wiesen nach, daß allein aufgrund der akustischen Information aus zwei Standardfragen bzw. aufgrund der sichtbaren Information aus 10 Sekunden Videoausschnitten der Zustand des Patienten nicht nur aus dessen eigenem Verhalten, sondern auch aus dem Verhalten des Interviewers erschlossen werden kann.

Offensichtlich kann selbst indirekt das Befinden des Patienten aufgrund nonverbaler Informationen des Interviewers bemerkt werden. Voraussetzung für eine solche Diskriminationsleistung der Beurteiler war, daß das Verhalten jeweils eines Interviewers in verschiedenen Situationen, d. h. bej unterschiedlichem Befinden desselben Patienten unmittelbar verglichen wurde. 
Intuitiv registriert man vielleicht diese Verhaltensinformation eines Individuums, d.h. also die idiosynkratischen Zusammenhänge zum Befinden, sofern man die Person in verschiedenen Zuständen oder Situationen erlebt hat.

Welche Zeit und wieviel Informationen notwendig sind, um solche Zusammenhänge zu bemerken, ist nicht bekannt. Beurteilungsstudien deuten darauf hin, daß hier sehr kurze Verhaltensstichproben ausreichen, um im intraindividuellen unmittelbaren Vergleich subtile Unterschiede valide zu entdecken. Innerhalb der ersten 3 min von 25 minütigen Interviews bemerken z. B. Psychiater die Hälfte der später insgesamt festgestellten Symptome (SANDIFER et al. 1970). Bereits innerhalb von 30 bis $60 \mathrm{~s}$ werden diagnostische Hypothesen gebildet (GAuron u. Dickinson 1969). Dabei besteht nur eine geringe Übereinstimmung zwischen vermuteter Wichtigkeit und dem tatsächlichen Beitrag der Informationen zur Diagnosenstellung. Offensichtlich spielen also zeitliche Aspekte und konsekutive Verarbeitung von Informationen bei der Urteilsbildung aufgrund komplexer Verhaltensinformationen eine bedeutsame Rolle.

$\mathrm{Ob}$ Verhalten als spezifischer Indikator für die Inferenz affektiver Zustände nutzbar ist, wie dies im unmittelbaren Vergleich von Videoaufzeichnungen oder bei einer längeren Zeit der Bekanntschaft der Fall ist, hängt wahrscheinlich auch davon ab, unter welchen unterschiedlichen Bedingungen das Verhalten beim Individuum beobachtet werden konnte. Der Kliniker oder der Angehörige kennen die Person, erleben also die spezifischen Veränderungen. Ihnen stünden somit auch, anders als einer fremden Person, die spezifischen Indikatoren als Inferenzbasis zur Verfügung.

Der Versuch, idiographische Elemente in der Diagnostik zu verwenden, zumindest wenn man nonverbales Verhalten als Informationsquelle heranzieht, würde den hier gefundenen individuellen Zusammenhängen von Verhalten und Befinden Rechnung tragen.

Nach den vorangehenden Erörterungen ist zudem ein intraindividueller Vergleich über den Verlauf wesentlich, um nonverbale Verhaltensinformationen nutzen zu können.

\section{Folgerungen für die Analyse kommunikativen Verhaltens}

Welche Strategien sind einzuschlagen, will man sozialkommunikative Verhaltensweisen als Informationsquelle für hirnorganische Syndrome in systematischer Weise erschlieBen?

1. Nach den klinischen Beschreibungen liegen genügend Hinweise vor, die eine gezielte Beobachtung mittels systematischer Verfahren ermöglichen. Solche Verfahren sind mit den technischen Möglichkeiten von Videoaufzeichnungen und systematischen Beobachtungsverfahren verfügbar.

2. Die systematische Beobachtung nonverbalen Verhaltens ist aufwendig, wahrscheinlich aber nicht aufwendiger als andere Analyseverfahren. In jüngerer Zeit wurden verschiedene brauchbare Methoden entwickelt, die genaue und reliable Beschreibungen des Verhaltens erlauben (s. SCHERER u. EKMAN 1982). Der Vorzug liegt in dem nichtinvasiven, unmittelbaren Zugang zu affektiven Vorgängen.

3. Die Leistung bei der Wahrnehmung und Verarbeitung affektiv getönten Materials, wie sie z. B. in Untersuchungen zum Gesichter-Erkennen erfaßt wurde, ist ebenso zu berücksichtigen wie die Aspekte der Produktion sozialer Signale. Letztere sind zwar 
schwieriger zu bestimmen und quantitativ zu beschreiben, sie sind aber besonders wichtig, um mögliche Dissoziationen von Emotionen und deren Ausdruck zu erfassen.

4. Im Hinblick auf die Abgrenzung und Differenzierung innerhalb des diffusen hirnorganischen Psychosyndroms wäre es wichtig, im individuellen Fall die betroffenen Hirnstrukturen zu spezifizieren. Über die assoziierten Funktionen hinaus sind die Beziehungen zur allgemeinen und spezifischen mimischen Aktivität einerseits und/oder der Affektsteuerung andererseits zu berücksichtigen. Damit hängt die Frage zusammen, inwieweit die Willkür- oder die Spontan/Reaktiv-Mimik durch diffuse oder lokale hirnorganische Läsionen betroffen ist.

5. Zu differenzieren ist zwischen Affekten als psychischem Erleben einerseits und Verhalten als Ausdruck dieser Affekte andererseits. Bisher läßt sich nur vermuten, auf welchen Verhaltensinformationen z. B. der Eindruck der Affektstarre oder Affektinkontinenz beruht. Die Diskrepanz von Affekterleben und der Unfähigkeit des Affektausdrucks bei der Parkinson-Erkrankung macht die Notwendigkeit dieser Differenzierung besonders deutlich.

Berücksichtigt man das nonverbale Verhalten als Informationsquelle über affektive und appetitive Zustände, so sind damit nicht-invasive Methoden verfügbar, die differenziert das Erleben abbilden können. Der Kliniker, der diese Informationen eher intuitiv nutzt, kann wahrscheinlich von den individuellen Komponenten des Ausdrucks abstrahieren und nimmt so das Symptom für das Syndrom.

Nach den meist auf intuitivem Eindruck beruhenden Beschreibungen erscheint es jedenfalls erfolgversprechend, nicht nur das Ausdruckserkennen, sondern auch das Ausdrucksverhalten selbst bei hirnorganischen Störungen systematisch zu erfassen und zu quantifizieren. Daß sich darin Veränderungen des affektiven Zustands individuenspezifisch abbilden lassen, wurde anhand von Verlaufsanalysen bei depressiven Patienten gezeigt.

Zusammen mit Informationen zum subjektiven Befinden und zu aktuellen Emotionen sind Aussagen über das Verhältnis von erlebtem und ausgedrücktem Affekt, aber auch Störungen im Affektsystem und, möglicherweise unabhängig davon, im Ausdruckssystem präziser zu treffen.

\section{Literatur}

AKISKAL HS (1979) A biobehavioral approach to depression. In: DepUe RA (ed) The psychobiology of depressive disorders. Academic Press, New York, pp 409-437

Avarello M (1983) Nonverbales Verhalten in klinisch-diagnostischen Gesprächssituationen - Eine Eindrucksstudie. Dissertation, Leopold-Franzens-Universität, Innsbruck

BENTE D, WIESER S (1953) Motorische Schablonen bei stufenweiser cerebraler Restitution. Psychiat Neurol 1: 13-18

Birkmayer W, Riederer P (1980) Die Parkinson-Krankheit. Biochemie, Klinik, Therapie. Springer, Wien

BLAzER DG (1982) Depression in later life. Mosby, St. Louis

BLEuLer E (1969) Lehrbuch der Psychiatrie, 11. Aufl. Springer, Berlin Heidelberg New York

Borod JC, CARON HS (1980) Facedness and emotion related to lateral dominance, sex and expression type. Neuropsychol 18:237-241

BRUYER R (1979) Le cerveau et la reconnaissance visuelle du visage humain. Acta Psychiat Beig 79: $113-143$ 
BRUYeR R (1980) Perception du visage humain et différences cérébrales hemisphériques chez le sujet normal. Année Psychol 80: 631-653

BRUYER R (1981) Asymmetry of facial expression in brain damaged subjects. Neuropsychol 19: 615-624

Bruyer R, Velge V (1980) Lésions cérébrales et reconnaissance visuelle du visage humain: Une étude préliminaire. Psychol Belg 20: 125-139

CAMPBell R (1978) Asymmetries in interpreting and expressing a posed facial expression. Cortex 14: $327-342$

CAPORAEL LR (1981) The paralanguage of caregiving: Baby talk to the institutionalized aged. J Pers Soc Psychol 40: 876-884

Cicone M, WaPNer W, Gardner H (1980) Sensitivity to emotional expressions and situations in organic patients. Cortex 16:145-158

EKMAN P, Friesen WV (1969) The repertoire of nonverbal behavior - Categories, origins, usage, and coding. Semiotica $1: 49-98$

Ekman P, Friesen WV (1978) Manual for the facial action code. Consulting Psychologist Press, Palo Alto

ELLGRING H (1981) Psychische Beanspruchung durch Sprache und Blickzuwendung in Gesprächsund Leistungssituationen. In: TENT L (Hrsg) Erkennen - Wollen - Handeln. Beiträge zur Allgemeinen und Angewandten Psychologie. Hogrefe, Göttingen, S276-290

Ellgring H, Derbolowsky J, von Dewitz A, Hieke S (1978) Standardisiertes Interview zum Verlauf depressiver Erkrankungen. Max-Planck-Institut für Psychiatrie, München

Fisch HU, Frey S, HirSBRUNNER HP (1983) Analyzing nonverbal behavior in depression. J Abnorm Psychol 92: 307-318

FOLSTEIN MF, LURIA R (1973) Reliability, validity, and clinical applications of the visual analogue mood scale. Psychol Med 3: 479-486

Gainotti G, Lemmo M (1976) Comprehension of symbolic gestures in aphasia. Brain Lang 3: $451-460$

Gauron EF, Dickinson JK (1969) The influence of seeing the patient first on diagnostic decision making in psychiatry. Am J Psychiat 126: 199-205

GerstenBRAND F (1967) Das traumatische apallische Syndrom. Springer, Wien

Goldblum MC (1980) La reconnaissance des expressions faciales émotionelles et conventionelles au cours des lésions corticales. Revue Neurol 136:711-719

GoOdglass H, KaPlaN E (1963) Disturbance of gesture and pantomime in aphasia. Brain 86: $703-720$

Heimann H, Spoerri T (1957) Das Ausdruckssyndrom der mimischen Desintegrierung bei chronisch Schizophrenen. Schweiz med Wschrft 35: 1126

HJORTSJOE C-H (1970) Man's face and mimic language. Nordens Boktryckeri, Malmö

JONES JH, PANSA M (1979) Some nonverbal aspects of depression and schizophrenia occurring during the interview. J Nerv Ment Disease 167: 402-409

KENDON A (1967) Some functions of gaze direction in social interaction. Acta Psychol 26: 22-63

KolB B, MiLner B (1981) Observations on spontaneous facial expression after focal cerebral excisions and after intracarotid injection of sodium amytal. Neuropsychol 19: 505-514

Kuracz J, Feldmar GA, Werner W (1979) Prosope-affective agnosia associated with chronic organic brain syndrome. J Am Geriatr Soc 27: 91-95

Leehey S, Carey SD, Diamond R, Cahn A (1978) Upright and inverted faces: The right hemisphere knows the difference. Cortex 14: 411-419

Leyhausen P (1967) Biologie von Ausdruck und Eindruck. Psychol Forsch 31: 113-176

MATARAZzo JD, SASLOW G (1961) Difference in interview interaction behavior among normal and deviant groups. In: BERG IA, BASS BM (eds) Conformity and deviation. Harper \& Row, New York, pp 286-327

PatTAY S (1982) Stimmungsbeeinflussung und Stimmausdruck. - Läßt sich die Befindlichkeit des Patienten aus der Stimme des Therapeuten erkennen? Diplomarbeit, Ludwig-Maximilians-Universität, München

Ploog D (1964) Verhaltensforschung und Psychiatrie. In: Gruhle HW, Jung R, Mayer-Gross W, MÖller M (Hrsg) Psychiatrie der Gegenwart, Bd. I/1B. Springer, Berlin Heidelberg New York, S.291-443

Ploog D (1980a) Verhaltensbiologische Ansätze zur Depressionsforschung. In: HeImANN H, GiedKE H (Hrsg) Neue Perspektiven in der Depressionsforschung. Huber, Bern, S 18-26 
Ploog D (1980b) Soziobiologie der Primaten. In: Kusker KP, Meyer Je, Moller C, Strömgen E (Hrsg) Psychiatrie der Gegenwart, Bd. I/2. Springer, Berlin Heidelberg New York, S 379-544

PLoog D (1980 c) Der Ausdruck der Gemütsbewegungen bei Mensch und Tieren. In: Max-PlanckGesellschaft (Hrsg), Jahrbuch 1980. Vandenhoek \& Ruprecht, Göttingen, S 66-97

Ploog D (1980d) Emotionen als Produkte des limbischen Systems. Med Psychol 6: 7-19

Poeck K (1969) Pathophysiology of emotional disorders associated with brain damage. In: VINKEN PJ, BRUYN GW (eds) Handbook of clinical neurology, vol 3. Disorders of higher nervous activity. Wiley, New York, pp 343-367

RAPACZYNSKI W, EhrLICHMAN H (1979) Opposite visual hemifield superiorities in face recognition as a function of cognitive style. Neuropsychol 17:645-652

ReNFORdT E, Busch H (1978) Quantifizierende Beurteilung des psychopathologischen Längsschnittprofils mit Hilfe audiovisueller Aufzeichnungen. Arzneimittel-Forschung: Drug Research 28: $1286-1288$

RUTTER DR, STEPHENSON GM (1972) Visual interaction in a group of schizophrenic and depressive patients: A follow-up study. Br J Soc Clin Psychol 11:410-411

SACKeim HA, GuR RC (1978) Lateral asymmetry in intensity of emotional expression. Neuropsychol 16: 473-481

SACKeIM HA, GuR RC, SAUCY MC (1978) Emotions are expressed more intensely on the left side of the face. Sci 202: 434-436

SAndifer MG, Hordorn A, Green LM (1970) The psychiatric interview: The impact of the first three minutes. Am J Psychiat 126: 968-973

SCHERER KR, EKMAN P (1982) (eds) Handbook of methods in nonverbal behavior research. Cambridge University Press, Cambridge

Schwarz D, Strian F (1972) Psychometrische Untersuchungen zur Befindlichkeit psychiatrischer und intern-medizinischer Patienten. Arch Psychiat Nervenkrankh 216: 70-81

Selby G (1968) Parkinson's disease. In: Vinken PJ, BruYn GW (eds) Handbook of clinical neurology, vol 6. Diseases of the basal ganglia. Wiley, New York, pp 173-211

Sergent J, BindRa D (1981) Differential hemispheric processing of faces: Methodological considerations and reinterpretation. Psychol Bull 89:541-554

Steinhausen D, Langer K (1977) Clusteranalyse - Einführung in Methoden und Verfahren der automatischen Klassifikation. De Gruyter, Berlin

Tölle R (1982) Psychiatrie, 6. Aufl. Springer, Berlin Heidelberg New York

Tomkins SS (1962) Affect, imagery, and consciousness. Vol I. The positive affects. Springer, New York

VARNEY N (1978) Linguistic correlates of pantomime recognition in aphasics. J Neurol Neurosurg Psychiat 41: 564- 568

WALLBOTT HG (1982) Bewegungsstil und Bewegungsqualität: Untersuchungen zum Ausdruck und Eindruck gestischen Verhaltens (in Vorbereitung). Beltz, Weinheim

Williams D (1969) Temporal lobe syndromes. In: VinkEN PJ, BruYN GW (eds) Handbook of clinical neurology, vol 2. Localization in clinical neurology. Wiley, New York, pp 700-724

WILSON SA (1924) Some problems in neurology: No. II - Pathological laughing and crying. J Neurol Psychopath 4: 299-333

WOLFF W (1943) The expression of personality. Harper, New York

Young AW, BIon PJ (1980) Absence of any developmental trend in right hemisphere superiority for face recognition. Cortex $16: 213-221$

ZüLCH KJ (1951) Traumatische Störungen der Motorik und Sensibilität und ihre Restitution. D Z Nervenheilk 166: 400-430 